\title{
Origen y perspectivas de las políticas de la Educación Intercultural Bilingüe en el Perú: utopía hacia una ElB de calidad
}

Artículo de investigación https://doi.org/ 10.19053/01227238.10831

Historia del artículo:

Recibido: 11/11/2019

Evaluado: 29/12/2020

Aprobado: 20/01/2021

Cómo citar este artículo:

Ruelas Vargas David "Origen y perspectivas de las políticas de la Educación Intercultural Bilingüe en el Perú: utopía hacia una EIB de calidad" Revista Historia de la Educación Latinoamericana vol.23 no.36 (2021)
David Ruelas Vargas'

Universidad Nacional del Altiplano Puno, Perú https://orcid.org/0000-0003-1315-5493

\section{Resumen}

Objetivo: de la investigación es contribuir al estudio del origen y perspectivas de las políticas de Educación Intercultural Bilingüe en el Perú, que busca atender a los pueblos originarios como medida pedagógica compensatoria a la implantación en las escuelas de la castellanización, lo que ocasionó dificultades en el aprendizaje de niños y niñas de pueblos indígenas. En el Perú, un país pluriétnico, multicultural y plurilingüe en donde coexisten diversos pueblos andinos, amazónicos y costeños, el Ministerio de Educación del Perú ${ }^{2}$ reconoce la existencia de 43 lenguas originarias en la amazonía y 4 en los Andes.

Originalidad / aporte: consiste en abordar y analizar el desarrollo e implementación de las políticas EIB en el sistema educativo peruano.

1 Doctor en Educación, magíster en Didáctica de las Ciencias Sociales, docente de la Facultad de Ciencias de la Educación - Universidad Nacional del Altiplano - Puno.divadrv@hotmail.com - ruelasvargasdavid@gmail. com

2 Ministerio de Educación, Documento nacional de Lenguas originarias del Perú (Lima: MINEDU, 2013). https://centroderecursos.cultura.pe/sites/ default/files/rb/pdf/Documento\%20Nacional\%20de\%20Lenguas $\% 20$ Originarias\%20del\%20Peru.pdf 
Método: aplicado es histórico y se soportó con la técnica de biblioteca y análisis e interpretación de documentos históricos de fuentes primaria y secundaria,

Estrategia de recolección de información: a partir de análisis de textos, artículos, archivos y revistas.

Conclusión: las políticas de educación bilingüe de los siglos XX y XXI han estado mediadas por la globalización y los organismos transnacionales como el Fondo Monetario Internacional, Banco Mundial, Banco Interamericano de Desarrollo - Washington, los pueblos indígenas no están libres de su influencia. Por la diversidad etnolingüística y pluricultural en la educación peruana del siglo XXI, las lenguas son prioridad como medio válido de aprendizaje por ser poseedoras de saberes y conocimientos. La educación bilingüe de calidad para los pueblos originarios sigue siendo una utopía.

Palabras clave: aprendizaje; cultura; diversidad;educación intercultural; lengua originaria.

\title{
Origin and perspectives of Intercultural Bilingual Education policies in Peru: utopia towards a quality IBE
}

\begin{abstract}
Objective: of the research is to contribute to the study of the origin and perspectives of Intercultural Bilingual Education policies in Peru, which seeks to serve native peoples as a compensatory pedagogical measure for the implementation in schools of Castilianization, which caused difficulties in the learning of children from indigenous peoples. In Peru, a multiethnic, multicultural and multilingual country where various Andean, Amazonian and coastal peoples coexist, the Ministry of Education of Peru recognizes the existence of 43 native languages in the Amazon and 4 in the Andes.
\end{abstract}

Originality/support: consists of addressing and analyzing the development and implementation of IBE policies in the Peruvian educational system.

Method: applied is historical and was supported with the library technique and analysis and interpretation of historical documents from primary and secondary sources, the information collection

Strategy/ information gathering: was based on the analysis of texts, articles, archives and magazines.

Conclusions: bilingual education policies of the 20th and 21st centuries have been mediated by globalization and transnational organizations such as 
the International Monetary Fund, World Bank, Inter-American Development Bank - Washington, indigenous peoples are not free from their influence. Due to the ethnolinguistic and multicultural diversity in Peruvian education in the 21 st century, languages are a priority as a valid means of learning because they possess knowledge and knowledge. Quality bilingual education for indigenous peoples remains a utopia.

Keywords: learnin;, culture; diversity; intercultural education; native language.

\section{Origem e perspectivas das políticas de Educação Intercultural Bilíngue no Peru: utopia para um IBE de qualidade}

\section{Resumo}

Objetivo: da pesquisa é contribuir para o estudo da origem e perspectivas das políticas de Educação Intercultural Bilíngue no Peru, que busca servir aos povos indígenas como medida pedagógica compensatória para a implantação em escolas de Castilianização, o que causou dificuldades no aprendizagem de filhos de povos indígenas. No Peru, país multiétnico, multicultural e multilíngue onde convivem diversos povos andinos, amazônicos e costeiros, o Ministério da Educação do Peru reconhece a existência de 43 línguas nativas na Amazônia e 4 nos Andes.

Originalidade/aporte: consiste em abordar e analisar o desenvolvimento e implementação de políticas de IBE no sistema educacional peruano.

Método; aplicada é histórica e apoiada na técnica de biblioteca e análise e interpretação de documentos históricos de fontes primárias e secundárias.

Estratégia/coleta de dados: baseou-se na análise de textos, artigos, arquivos e revistas. Conclusões: as políticas de educação bilíngue dos séculos 20 e 21 foram mediadas pela globalização e por organizações transnacionais como o Fundo Monetário Internacional, Banco Mundial, Banco Interamericano de Desenvolvimento - Washington, os povos indígenas não estão livres de sua influência. Devido à diversidade etnolinguística e multicultural da educação peruana no século XXI, as línguas são prioritárias como meio válido de aprendizagem por possuírem conhecimentos e saberes. Educação bilíngue de qualidade para povos indígenas continua sendo uma utopia.

Palavras-chave: aprendizagem; cultura; diversidade; educação intercultural; língua nativa. 


\section{Introducción}

El Perú es un país pluriétnico, multicultural y plurilingüe, cuya heterogeneidad se manifiesta en la coexistencia de diversos pueblos andinos, amazónicos y costeños. Es un país que posee:

(...) una diversidad étnica, social, cultural y biológica que determina diversos modos de ser, de hablar, de sentir, de pensar. (...) según el lingüista Gustavo Solís en el Perú existen en total 43 lenguas, incluidas tres de la zona andina. Históricamente la costa también tuvo una gran diversidad cultural que aún hoy se mantiene viva en algunas zonas de la franja costera. ${ }^{3}$

La Educación Intercultural Bilingüe (EIB) y la Educación Bilingüe Intercultural EBI "expresan diferentes matices de un proceso emancipatorio de la educación bilingüe enfocado principalmente en el uso instrumental de la lengua indígena en el proceso educativo, desde mediados de los años 30 del siglo XX"4. El Estado peruano no contaba con un sistema educativo bilingüe para los niños y niñas indígenas de los sectores rurales o áreas andinas y amazónicas ${ }^{5}$ la población indígena era vista como "ignorante" y mentalmente limitada 6 . Hasta mediados del siglo XX, las políticas educativas peruanas aspiraban a generar el aprendizaje de una y en una sola lengua: el castellano, es decir, a la uniformización idiomática ${ }^{7}$.

El panorama lingüístico cambió notablemente en la segunda mitad del siglo pasado. Se sabe que en 1940 más de la mitad de peruanos sabía una lengua indígena (el 50 \% conocía tan solo el quechua). Hasta hace dos o tres décadas, en la amazonía se contaban más de 56 lenguas y alrededor de 12 o 13 familias lingüisticas, aunque siempre se reconocía que no eran cifras exactas. Estudios recientes permiten una mejor clasificación de las lenguas y, lamentablemente, dan también cuenta de la pérdida de muchas de ellas ${ }^{8}$. Durante muchos siglos, estas lenguas originarias han sido consideradas como dialectos y se les ha dado un "estatus inferior" al del castellano. Asimismo, se afirmaba que estos "dialectos" (lenguas) de Perú no tenían gramáticas, sin embargo, las teorías lingüísticas nos han permitido desmentir ese postulado. Las diversas investigaciones sobre las lenguas del Perú y del mundo, en las últimas décadas, han permitido analizarlas y clasificarlas en familias lingüísticas?.

3 Ministerio de Educación. Hacia una Educación Intercultural Bilingüe de Calidad (Lima: MINEDU, 2013), 14. http:// www.minedu.gob.pe/minedu/archivos/a/002/01-general/2-propuesta_pedaggogica_eib_2013.pdf

4 Teresa Valiente-Catter. "La educación intercultural bilingüe en la encrucijada global. Currículo intercultural y aprendizaje multicultural", Alteridad. Revista de Educación vol. 3, n. 2 (2008): 25.

5 David Ruelas Vargas. "Los movimientos indígenas y la educación del siglo XX en el sur andino puneño peruano", Revista Historia de la Educación Latinoamericana vol. 21, n. 33 (2019): 64. https://doi.org/10.19053/01227238.9354.

6 Jorge Alberto Ccahuana Córdova. "Según la capacidad intelectual de cada uno': élites, Estado y educación indígena a inicios del siglo XX" (tesis de licenciatura en Historia, Pontificia Universidad Católica del Perú, 2013), 19.

7 David Eduardo Paredes et al., "Diagnóstico de la Educación Intercultural Bilingüe (EIB) en el Perú". Informe de la Universidad de San Martín de Porres, Perú, 2017. https://usmp.edu.pe/idp/wp-content/uploads/2016/05/diagnstico_de_eib_en_el_per.pdf (30 de marzo, 2020).

8 Madeleine Zúñiga Castillo. La Educación Intercultural Bilingüe: el caso peruano (Buenos Aires: Foro Latinoamericano de Políticas Públicas - FLAPE, 2008), 16.

9 Ministerio de Educación, Documento nacional de Lenguas originarias, óp. cit., 12. 


\begin{abstract}
Perú hacia el bicentenario es un (... ) país que contempla una vasta diversidad etno-lingüística, cuyas formas de existencia cultural se ven abonadas entre los temas más álgidos y críticos como proyecto-país. Los datos del Ministerio de Cultura refieren que en Perú prexiste 47 lenguas indígenas originarias, siendo Loreto la región de mayor diversidad lingüística (27); asimismo, la región Cusco, sigue siendo la región de primer orden de hablantes en lengua originaria, simultáneamente, si a este se le incluyen las regiones de Apurímac, Huancavelica y Ayacucho, hacen una proporción del 50 \% de la población de expresión quechua; además, desde el sur del país, Puno, se perfila como la región que concentra las tres cuartas partes de la demografía con lengua materna aimara, seguido por Tacna (10 \%), Lima/Callao (7 \%), Arequipa (4\%) y Moquegua (4\%). ${ }^{10}$
\end{abstract}

A pesar del crecimiento económico ocurrido en la última década, el Perú sigue siendo un país con altos índices de pobreza y de desigualdad educativa que afecta a los sectores más vulnerables: los pueblos indígenas andinos, amazónicos y costeños. Pareciera que, por un lado, la mano invisible del mercado no puede corregir esta situación, y, por el otro, que persiste una discriminación institucionalizada contra la población indígena que se ha sedimentado a través de las políticas públicas a lo largo del periodo republicano de nuestra historia $^{11}$, en la población indígena peruana se concentra la mayor desigualdad económica y social. La incidencia de pobreza total es de 1,8 veces mayor (33,39 \%) que la registrada en la población cuya lengua materna es el castellano (18,77 \%). Según la autopercepción del origen étnico, se tiene que la población de origen nativo enfrenta una incidencia de la pobreza del 26,21 \%, mientras que el porcentaje en la sierra es de $29,88 \%$ y en la selva $27,52 \%{ }^{12}$.

Durante la década de 1980 surge en América Latina la Educación Bilingüe Intercultural (EBI), un movimiento positivo en el reconocimiento de la pluralidad lingüístico-cultural por tener el propósito de consolidar y generalizar ese valor que en definitiva contribuirá en la formación de auténticas sociedades multiculturales e interculturales ${ }^{13}$, y es así que en el discurso educativo latinoamericano surgen siglas como las de EB (Educación Bilingüe), EBB (Educación Bilingüe Bicultural), EBI (Educación Bilingüe Intercultural) y, más recientemente, EIB (Educación Intercultural Bilingüe), para referirse a nuevos enfoques y modalidades educativas. Estas se han orientado a atender a la niñez y adultos analfabetos indígenas, en aras de propiciar una educación básicamente centrada en la tolerancia y el respeto a la pluralidad cultural ${ }^{14}$. La importancia de la investigación se debe a que enfocó su objetivo principal en estudiar el origen y las perspectivas de las políticas de Educación Intercultural Bilingüe en el Perú, lo cual ofrecerá datos sobre el desarrollo, políticas y resultados de la EIB hacia el bicentenario del Estado peruano. La investigación presenta el análisis, interpretación

10 Franklin Canaza-Choque y Jesús Huanca-Arohuanca. "Perú 2018: hacia una Educación Intercultural Bilingüe sentipensante", Sciéndo. Ciencia para el desarrollo vol. 21, n. ${ }^{\circ} 4$ (2018): 516.

11 Enrique Vásquez Huamán et al., Niñez indígena y Educación Intercultural Bilingüe en el Perú: Estadísticas recientes, preguntas (i)resueltas y tareas pendientes (Lima: Care Perú, 2009), 5.

12 Instituto Nacional de Estadística e Informática, "Evolución de la pobreza monetaria 2009-2014. Informe Técnico", Lima, 2015, 61.

13 Melchor Huamán Cosi. Educación Bilingüe Intercultural (EBI) en el Perú, siglo XXI (Perú: Lanic, 2007). http://lanic. utexas.edu/project/etext/lilas/ilassa/2008/cosi.pdf (30 de marzo, 2020)

14 Ynty Koyllor Martínez Alpaca. "El enfoque del Estado en las políticas educativas interculturales bilingües: Los planes educativos en el Perú, 2005-2008” (tesis de maestría en Política Social, Universidad Nacional Mayor de San Marcos, 2012), 42. 
y descripción del problema planteado, para generar análisis de las políticas educativas actuales en cuanto a la educación bilingüe de los aimaras, quechuas y amazónicos.

La investigación es de método histórico y como técnica de biblioteca con análisis de documentos históricos con bases de fuentes primaria y secundaria (libros, revistas, artículos científicos, tesis, periódicos e informes). Desde el punto de vista teórico es relevante porque se demuestran políticas educativas hacia una educación bilingüe para los pueblos originarios del Estado peruano a mediados del siglo XX y XXI.

\section{Figura 1. Familias etnolingüísticas en el Perú}

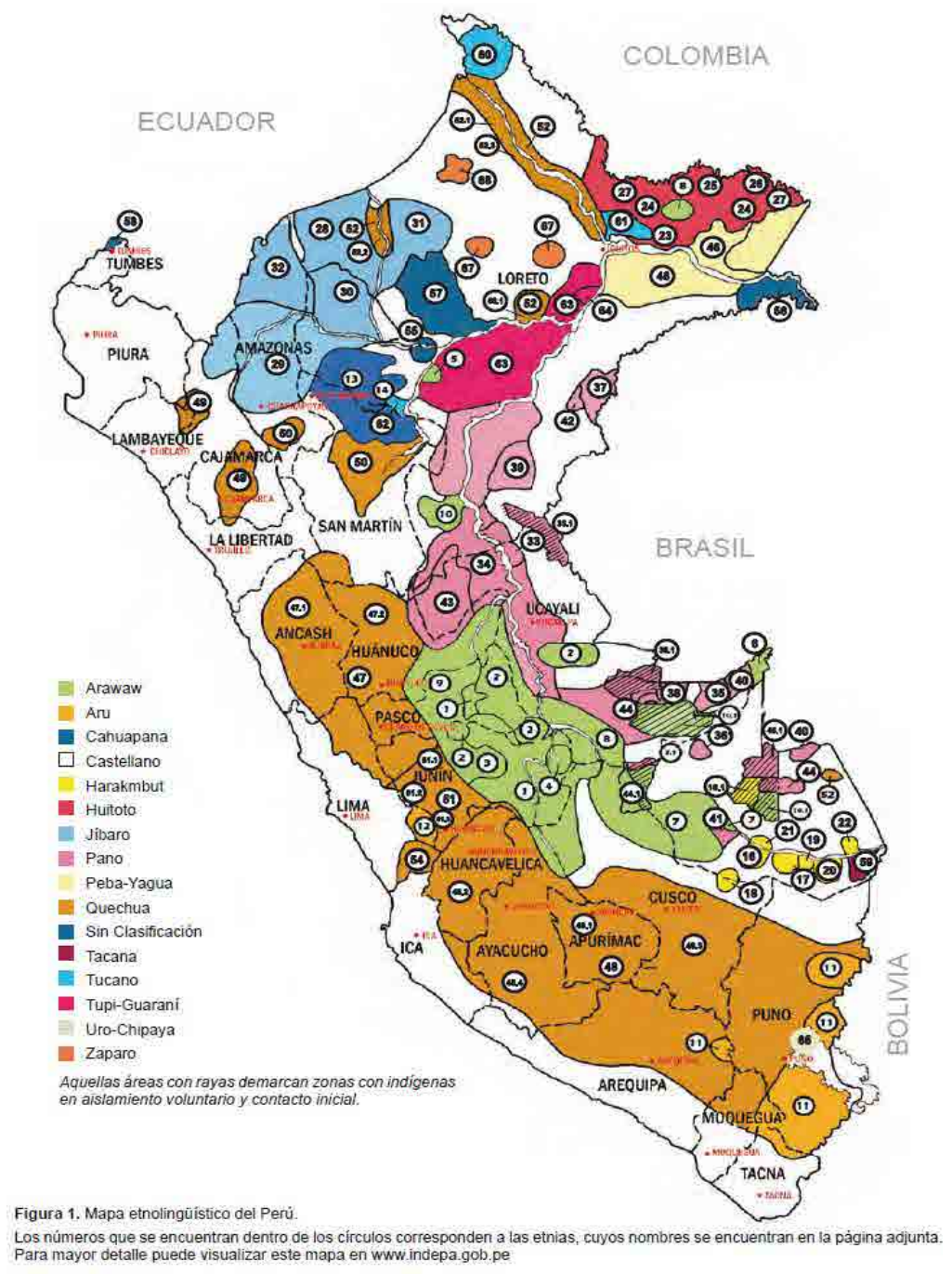

Fuente: INDEPA ${ }^{15}$.

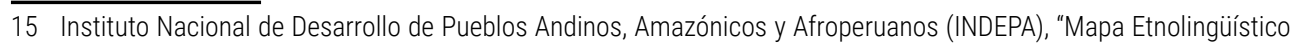
del Perú", Revista Peruana de Medicina Experimental y Salud Pública vol. 27, n. ${ }^{\circ} 2$ (2010): 290. 


\section{Material y método de estudio}

El diseño de investigación es histórico, nos permite estudiar los hechos del pasado con el fin de encontrar explicaciones causales a las manifestaciones propias de las sociedades actuales. Este tipo de investigación busca reconstruir el pasado de la manera más objetiva y exacta posible, para lo cual, de forma sistemática, recolecta, evalúa, verifica y sintetiza evidencias que permiten obtener conclusiones válidas, a menudo derivadas de hipótesis ${ }^{16}$.

La investigación de análisis documental consiste en la exploración de minutas, revistas, reportes, software, políticas y planes curriculares, fotografías, discursos, reportes, registros, libros, capítulos, artículos académicos, ensayos de posición, notas, manuscritos, tesis, etc., académicos e inéditos (a menudo disponibles en internet), cartas, revistas y diarios ${ }^{17}$. El análisis documental es una forma de investigación técnica, un conjunto de operaciones intelectuales que busca describir y representar los documentos de forma unificada y sistemática para facilitar su recuperación. Comprende el procesamiento analítico-sintético que, a su vez, incluye la descripción bibliográfica y general de la fuente, la clasificación, indización, anotación, extracción, traducción y la confección de reseñas ${ }^{18}$.

\section{Resultados y discusiones}

Antecedentes del accionar político de Educación Intercultural Bilingüe en Latinoamérica

Durante el siglo XX, en América Latina las poblaciones indígenas frecuentemente han exigido su derecho a la educación, distinguiéndose las siguientes etapas ${ }^{19}$ :

a) La primera etapa transcurre alrededor de los años de 1930. Lo más antiguo es de México, donde surge la educación bilingüe como alternativa a las políticas de castellanización del gobierno.

b) Segunda etapa: de los años 1970, cuando surge un tipo de educación en función de los derechos de las lenguas indígenas y sus hablantes.

c) Tercera etapa: la expansión en la década de 1990 de la EIB en Latinoamérica es la más completa y reciente a la que hemos tenido acceso.

Distintos estudios al finalizar el siglo XX “(...) contabilizan en diecisiete países donde se ha desarrollado algún tipo de Educación Bilingüe: Argentina, Bolivia, Brasil, Colombia,

16 Mario Tamayo y Tamayo. Módulo 2. La investigación. Serie Aprender a investigar (Santa Fe de Bogotá: ICFES, 1998), 42.

17 Michele Knobel y Colin Lankshear. Maneras de saber: tres enfoques para la investigación educativa (Morelia, México: Universidad Pedagógica Nacional, 2003), 45.

18 María Elinor Dulzaides y Ana maría Molina. "Análisis documental y de información: dos componentes de un mismo proceso", Acimed vol. 12, n. ${ }^{2} 2$ (2004): 9.

19 Luis Enrique López. "La cuestión de la interculturalidad y la educación latinoamericana" (documento de apoyo, Séptima reunión del Comité Regional Intergubernamental del Proyecto Principal de Educación en América Latina y el Caribe, Cochabamba, 5 al 7 de marzo de 2001), 8. 
Costa Rica, Chile, Ecuador, Guatemala, Guyana Francesa, Honduras, México, Nicaragua, Panamá, Paraguay, Perú, Surinam y Venezuela"20. Desde los años de 1990 la Educación Intercultural Bilingüe se ha convertido en un tema de prioridad, que está presente en las políticas públicas y en las reformas educativas constitucionales ${ }^{21}$, la educación intercultural es un tema controvertido que atraviesa las luchas políticas, principalmente en Ecuador y en Bolivia, y que le presenta varios cuestionamientos a las teorías políticas de origen europeo a las que estamos acostumbrados a tener como referencia ${ }^{22}$.

\section{Figura 2. Desarrollo del accionar político de la educación bilingüe entre 1930 y 1990.}

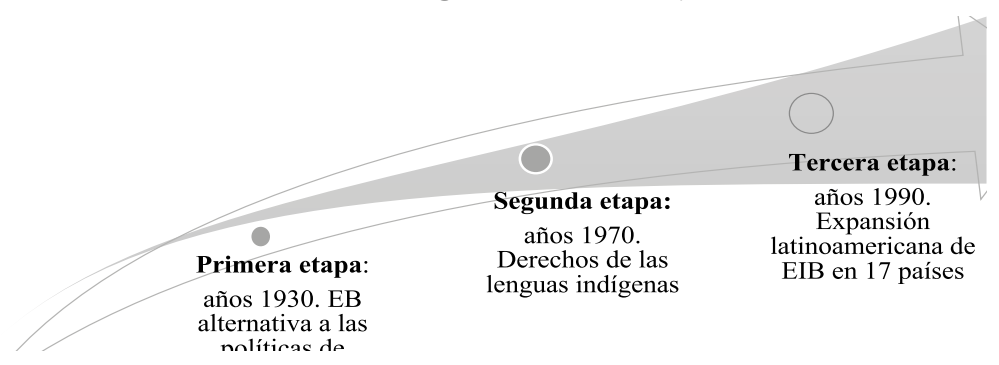

Fuente: elaboración propia.

El desarrollo de la Educación Intercultural Bilingüe en el continente tiene que ver con las experiencias de educación popular, realizadas a todo lo largo de América Latina, particularmente a partir de los años 196023; en este contexto dinámico se han desarrollado diversas experiencias de cooperación, destacables por su aporte a la EIB en las comunidades y en la formación de profesionales indígenas en la educación superior ${ }^{24}$, que nos enorgullece ser culturalmente diversos ${ }^{25}$, esto indica que los indígenas no solo deben concentrar sus esfuerzos por una educación para la preservación y promoción de su cultura, también deben tener una educación que les permita comprender y relacionarse con la cultura y la lengua del país en el cual habitan, ${ }^{26}$ respecto a la cultura, no debemos olvidar la importancia que esta tiene en la educación de muchas generaciones, a partir de la pertenencia a una cultura aprendemos muchos saberes, prácticas, tradiciones y estilos de vida, ${ }^{27}$ las sociedades indígenas desean que

20 Delia María Fajardo Salinas. "Educación intercultural bilingüe en Latinoamérica: un breve estado de la cuestión", LiminaR. Estudios sociales y humanísticos vol. IX, n. ${ }^{\circ} 2$ (2011): 21.

21 Catherine Walsh. "Interculturalidad crítica y educación intercultural”, en Construyendo Interculturalidad Crítica, eds. J. Viaña, L. Tapia y C. Walsh (La Paz: IICAB, 2010), 2.

22 Vera Maria Ferrão Candau. "Educación Intercultural en América Latina: distintas concepciones y tensiones actuales", Estudios Pedagógicos vol. 36, n. ${ }^{\circ} 2$ (2010): 336.

23 Ibíd.

24 Guillermo Williamson y Susana Navarrete, "Cooperación internacional y Educación Intercultural Bilingüe en Chile", Revista Mexicana de Investigación Educativa vol. 19, n. ${ }^{\circ} 60$ (2014): 24.

25 Sylvia Schmelkes. "Educación para un México intercultural”, Sinéctica, n. ${ }^{\circ} 40$ (2013): 3.

26 Yeny Alexandra Pulido, "La etnoeducación bilingüe: logro político y desafío para las etnias", Lenguaje vol. 40, n. ${ }^{\circ}$ (2012): 236.

27 Miriam Grimaldo Muchotrigo, "Identidad y política cultural en el Perú", Liberabit, n. ${ }^{12}$ (2006): 42. 
sus hijos e hijas se conviertan en ciudadanos, pero en ciudadanos indígenas interculturales que puedan vivir en un Estado que los respete y los valore en su diferencia étnica y cultural28.

Si bien a mediados del siglo XX la interculturalidad y la educación bilingüe predominantes en América Latina eran manipuladas por la clase política y el positivismo cientificista, tenemos claro que la práctica intercultural solo puede alcanzarse cuando la Educación Intercultural Bilingüe logre condiciones de simetría, tanto cultural como social, económica, etc. (cuando las distintas culturas sean capaces de coexistir en pie de igualdad). Por tanto, la interculturalidad va más allá del reconocimiento y la reivindicación de las diferencias culturales lingüísticas o la educación pertinente. La fuerza de la propuesta intercultural se dirige a una refundación de la nación peruana (refundar el Estado-nación, reconocer en él capacidades sociales, de diálogo intercultural que implique la convergencia y movilización de universos culturales y voluntades políticas muy diferentes), vinculando la afirmación de plurinacionalidad, con formas efectivas de ejercer ciudadanía de modo equitativo.

La globalización y los cambios ocurridos a nivel político, económico, social y cultural, en los últimos treinta años han delineado un nuevo escenario respecto de las políticas de Educación Intercultural Bilingüe en América Latina. La UNESCO sostenía el 2012 que los procesos de migraciones rurales y urbanas, el surgimiento de la etnicidad y la etnogénesis de los pueblos indígenas requerían de la promoción de una educación intercultural bilingüe, no solo como una forma de reparación histórica de los gobiernos a su permanente exclusión social, sino como una política internacional sustentada en las disposiciones de la Organización de Estados Iberoamericanos (1997), que sugieren la puesta en marcha de Políticas Gubernamentales Educativas para los Pueblos Indígenas en todas las naciones latinoamericanas. ${ }^{29}$

La globalización es un proceso que tiene muchos semblantes y que influye de manera determinante en todos los grupos y actores sociales; en este sentido, los pueblos indígenas no están libres de su influjo (poder que tienen las personas sobre la manera de pensar o actuar). El desafío para los pueblos indígenas es saber cómo enfrentarlo, e identificar cuáles son las dificultades y las oportunidades que tienen ante un fenómeno social, político, económico. La educación intercultural surge como la necesidad de lograr, desde la escuela y la comunidad, actitudes transformadoras en un ambiente de convivencia, de colaboración, tolerancia y el respeto ante la diversidad.

Proceso histórico de las políticas de la Educación Intercultural Bilingüe en el Perú

Un antecedente más cercano a las primeras políticas sobre educación bilingüe lo encontramos en la "Mesa Redonda sobre el Monolingüismo Quechua y Aimara y la Educación en el Perú", convocada en 1963 por José María Arguedas, entonces director de la Casa de la Cultura. Las acertadas recomendaciones emitidas en esa reunión por los estudiosos de las nacientes ciencias sociales en el país tardaron casi una década en plasmarse en la Política Nacional de Educación Bilingüe (PNEB) propuesta en 1972, la primera política en la región

28 Oscar Espinosa. "Los planes de vida y la política indígena en la Amazonía peruana", Anthropologica vol. 32, n. 32 (2014): 91.

29 Carlos Mondaca Rojas y Yeliza Gajardo Carvajal. "La Educación Intercultural Bilingüe en la región de Arica y Parinacota, 1980-2010", Diálogo Andino, n. ${ }^{4} 22$ (2013): 69. 
sudamericana que respondía a nuestra diversidad étnica, lingüística y cultural desde el sistema educativo nacional ${ }^{30}$.

Las políticas educativas desarrolladas en el Perú hasta la década de 1960 estaban dirigidas a castellanizar a los pueblos originarios, es decir, a la homogenización idiomática, políticas que fueron quebradas durante el gobierno de Juan Velasco en la década de 1970, cuando se pudieron distinguir las políticas educativas bilingües y la normatividad sobre el uso y manejo de las lenguas indígenas del castellano en la educación.

Si bien en el Perú ha habido, desde hace varias décadas, un reconocimiento oficial de la educación bilingüe para los pueblos indígenas, su institucionalización ha sido frágil y discontinua. Se pueden distinguir ciertas etapas (...):

a) La etapa que se inicia en 1972, con la Ley de Reforma Educativa (Decreto Ley 19326 del 21-3-1972), promulgada durante el gobierno del general Juan Velasco, en la cual se expresa la necesidad de una educación bilingüe. Sobre la base de esa ley, se formula una Política Nacional de Educación Bilingüe. Poco después se oficializa el quechua (Decreto Ley 21156 del 27-5-1975).

b) La etapa que corresponde a los años 1985-1989, durante la cual se da la Resolución Ministerial 1218-85-ED que oficializa el alfabeto quechua y aimara. En ese mismo periodo, se crea la Dirección General de Educación Bilingüe Intercultural (1987) que, años más tarde, queda desactivada.

c) La etapa que va de 1990 a 1995, en la que se establece una política de educación intercultural y de educación bilingüe, y durante la cual se promulga la nueva Constitución Política del Perú (1993) que, en su artículo n. ${ }^{\circ}$ 17, expresa que es obligación del Estado fomentar la educación bilingüe e intercultural, según las características de cada zona.

d) La etapa que va de 1996 a 2000, durante la cual se crea, en 1996, la Unidad de Educación Bilingüe Intercultural (UNEBI), dentro de la Dirección Nacional de Educación Inicial y Primaria. Se diseña un Plan Nacional de Educación Bilingüe Intercultural (1997-2000). Se desarrolla, de un modo más sistemático, un programa de educación bilingüe intercultural destinado a niños de pueblos indígenas andinos y amazónicos (...). ${ }^{31}$

En 2001 se constituye un Comité Consultivo Nacional de la Educación Bilingüe Intercultural (Resolución Ministerial n. ${ }^{\circ}$ 235-2001-ED), se realizan consultas nacionales que conducen a la definición de un documento sobre Política Nacional de Lenguas y Culturas en la Educación. Se elabora, finalmente, un Plan Estratégico 2001-2005 para el futuro desarrollo tanto de la educación intercultural como de la Educación Bilingüe Intercultural.

30 Zúñiga Castillo. La Educación Intercultural Bilingüe, 46.

31 Juan Carlos Godenzzi. "La educación bilingüe intercultural en el Perú", Lexis vol. XXV, n. ${ }^{\text {s }} 1$ y 2 (2001): 300. 
En el año 2002 el Estado peruano reconoce la diversidad cultural peruana como un valor y fomenta la educación bilingüe intercultural en las regiones donde habitan los pueblos indígenas, mediante la Ley n. ${ }^{\circ}$ 27818. Promueve en las instituciones educativas para los pueblos indígenas la incorporación, por nombramiento o contrato, de personal docente con dominio de la lengua originaria de la zona.

En el año 2018, mediante la Resolución Ministerial MINEDU 646-2018, se aprueba la Norma Técnica denominada "Disposiciones para el Registro de Instituciones Educativas que brindan el Servicio de Educación Intercultural Bilingüe" cuyo objetivo es establecer criterios para registrar a las Instituciones Educativas Públicas de Educación Básica y Programas No Escolarizados de Educación Inicial que implementan el Modelo de Servicio Educativo Intercultural Bilingüe y de los Centros de Educación Técnico.

Durante la primera fase del gobierno militar del general Juan Velasco Alvarado (19681975) se dieron significativas reformas para la educación: se implantó la educación inicial, se promulgó la Ley General de Educación 19326 (1972) y se creó la Política Nacional de Educación Bilingüe-Bicultural, que proponía introducir en la enseñanza aspectos culturales indígenas a partir de sus formas tradicionales (artesanía, constitución familiar, forma de organización social) ${ }^{32}$. La segunda fase del gobierno militar, asumida por el general Francisco Morales Bermúdez (1975-1980), difundió el documento "Lineamiento de la Política Educativa Nacional", con el objetivo de anular la reforma educativa hecha por su predecesor. A pesar de la falta de apoyo técnico y financiero del gobierno, la idea de una educación bilingüe para las poblaciones indígenas permaneció y diversos programas de educación bilingüe de la región andina y amazónica dieron continuidad a sus acciones.

En 1982, el gobierno civil de Fernando Belaúnde Terry retomó la política lingüística pro-lenguas indígenas, oficializada por la Constitución de 1979, que declara en el artículo 35: "El Estado promueve el estudio y conocimiento de las lenguas aborígenes. Garantiza el derecho de las comunidades nativas a recibir educación primaria, también, en su propio idioma y lengua"33.

Entre los años 1991 y 1995 se desarrollaron las políticas de Educación Intercultural Bilingüe del Estado peruano con la Unidad de Educación Bilingüe Intercultural (UNEBI), dependiente de la Dirección Nacional de Educación Inicial y Primaria ${ }^{34}$. Luego, en el año 2002, se formuló otra política educativa — esta vez elaborada por la Dirección de Educación Intercultural Bilingüe (DEIB) del Ministerio de Educación—, el Proyecto de Política Nacional de Lenguas y Culturas en la Educación, en el marco de la EIB. Creada la Dirección Nacional de Educación Bilingüe Intercultural (DINEBI), se logró la Ley Nacional de Lenguas, realizada en consulta con las organizaciones representativas de las lenguas y culturas del país, y la inclusión de la Educación Bilingüe Intercultural en el Proyecto de Reforma Constitucional ${ }^{35}$.

Aunque a principios del 2000 se dio inicio al diseño participativo de una Política Nacional de Lenguas y Culturas en la Educación, esta política no fue asumida por el Ejecutivo, y como

\footnotetext{
32 Huamán Cosi, Educación. Bilingüe Intercultural.

33 Asamblea Constituyente del Perú 1979, Constitución Política del Perú (Lima: ACP, 1979), 7.

34 Huamán Cosi, Educación Bilingüe Intercultural.

35 Grimaldo Muchotrigo, "Identidad y política cultural en el Perú", 44
} 
siguiente etapa la Dirección Nacional de Educación Bilingüe (DINEBI) decidió aprobarla en el año 2005 como "Lineamientos de Política de la Educación Bilingüe Intercultural", con Resolución Directoral 175-2005-ED. Por otro lado, en el año 2007 el Proyecto Educativo Nacional (PEN) lanza la propuesta de la educación que queremos en una proyección al 2021, incorporando la interculturalidad como eje transversal desde una perspectiva de educación intercultural para toda la población estudiantil del país ${ }^{36}$.

Figura 3. Desarrollo de las direcciones EIB en el Perú, 1995 - 2015.

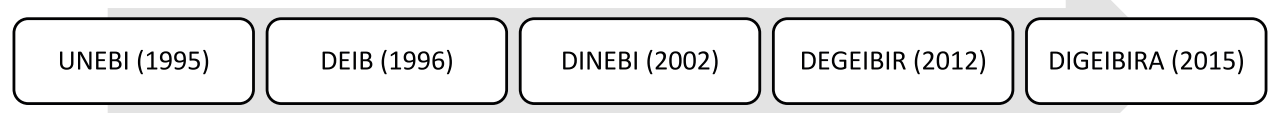

Fuente: elaboración propia.

Con la finalidad de implementar la educación intercultural, se crea en el año 2012 la Dirección General de Educación Intercultural, Bilingüe y Rural (DEGEIBIR), responsable de normar y orientar la política nacional de Educación Intercultural Bilingüe y Rural en las etapas, niveles, modalidades, ciclos y programas del sistema educativo nacional. Dependía del Viceministerio de Gestión Pedagógica, mismo que fue desactivado en el año 2015, de modo que en la actualidad las políticas de interculturalidad educativa las viene trabajando la Dirección General de Educación Básica Alternativa, Intercultural Bilingüe y de Servicios Educativos en el Ámbito Rural (DIGEIBIRA) (figura 2).

En términos generales, en los programas experimentales de educación bilingüe (EB) para poblaciones indígenas que nacieron y se extinguieron o renacieron en las décadas del setenta y ochenta, es más fuerte la conciencia de la diversidad y necesidades lingüísticas que las de carácter étnico-cultural. ${ }^{37}$

\section{Las lenguas originarias e instituciones educativas EIB en el Perú}

Con la promulgación de la Política Nacional de Educación Bilingüe (PNEB) de 1972, y el Decreto Ley n. ${ }^{\circ} 21156$ (que oficializó el quechua en 1975) el Perú se situó en la vanguardia de la legislación que promovió la educación bilingüe en América del Sur. El artículo 12 de la Ley General de Educación de 1972, declara:

La educación considerará en todas sus acciones la existencia en el país de diversas lenguas que son medios de comunicación y expresión de cultura, y velará por su preservación

36 Ministerio de Educación. Política sectorial de Educación Intercultural y Educación Intercultural Bilingüe (Lima: MINEDU, 2018), 34.

37 Madeleine Zúñiga Castillo y Juan Ansión Mallet. Interculturalidad y educación en el Perú (Lima: Foro Educativo 1997), 20. https://red. pucp.edu.pe/ridei/wp-content/uploads/biblioteca/inter59.PDF (02 de abril, 2020). 
y desarrollo. La castellanización de toda la población se hará respetando la personalidad cultural de los diversos grupos que conforman la sociedad nacional y utilizando sus lenguas como vínculo de educación. ${ }^{38}$

Al 2013, según el último registro del Ministerio de Cultura, en el Perú se reconoce la existencia de 55 pueblos originarios o indígenas que hablan 47 lenguas originarias, agrupadas en 19 familias lingüísticas (43 en la amazonía y cuatro en los Andes). A los niños, niñas, adolescentes, jóvenes y personas adultas mayores de estos pueblos se les debe ofrecer el servicio de Educación Intercultural y Bilingüe, que les garantice los aprendizajes que cada estudiante del Perú debe lograr, pero que a su vez considere los conocimientos, historias, prácticas y valores de su pueblo, así como el fortalecimiento y desarrollo de su lengua ${ }^{39}$. Según el Instituto Nacional de Estadística e Informática (INEI), la población que habla una lengua originaria y materna en el Perú asciende a 4045713 personas, estimándose que la cantidad de niños, niñas, adolescentes y jóvenes en edad de acceder a la educación básica (3 a 24 años) asciende a 1494253 personas, 744921 mujeres y 749332 varones.

\section{Cuadro n.o 1. Relación de lenguas originarias vigentes del Perú}

\begin{tabular}{|c|c|c|c|}
\hline Andinas & \multicolumn{3}{|c|}{ Amazónicas } \\
\hline $\begin{array}{c}\text { Aimara } \\
\text { Cauqui } \\
\text { Jaqaru } \\
\text { Quechua }\end{array}$ & $\begin{array}{l}\text { Achuar } \\
\text { Amahuaca } \\
\text { Arabela } \\
\text { Ashaninka } \\
\text { Awajún } \\
\text { Bora } \\
\text { Capanahua } \\
\text { Cashinahua } \\
\text { Chamicuro } \\
\text { Ese eja } \\
\text { Harakbut } \\
\text { Iñapari } \\
\text { Iquitu } \\
\text { Isconahua } \\
\text { Kakataibo }\end{array}$ & $\begin{array}{l}\text { Kakinte (Caquinte) } \\
\text { Kandozi-Chapra } \\
\text { Kukama-Kukamiria } \\
\text { Madija (Culina) } \\
\text { Maijuna } \\
\text { Matsigenka } \\
\text { Matses } \\
\text { Muniche } \\
\text { Murui-Muinani } \\
\text { Nanti } \\
\text { Nomatsigenga } \\
\text { Ocaina } \\
\text { Omagua } \\
\text { Resigaro } \\
\text { Secoya }\end{array}$ & $\begin{array}{l}\text { Sharanahua } \\
\text { Shawi } \\
\text { Shipibo-Konibo } \\
\text { Shiwilu } \\
\text { Taushiro } \\
\text { Tikuna (Ticuna) } \\
\text { Urarina } \\
\text { Wampis } \\
\text { Yagua } \\
\text { Yaminahua } \\
\text { Yanesha } \\
\text { Yine } \\
\text { Yora (Nahua) }\end{array}$ \\
\hline 4 lenguas & \multicolumn{3}{|l|}{43 lenguas } \\
\hline
\end{tabular}

Fuente: Ministerio de Educación, Documento Nacional de Lenguas Originarias, 16.

38 Ministerio de Educación, Política Nacional de Educación Bilingüe (Lima: MINEDU, 1972), 29.

39 Ministerio de Educación, Política sectorial de Educación Intercultural, 30. 
Estas lenguas se consideran vigentes porque tienen hablantes. Según resultados del Censo Nacional XII de Población y VII de Vivienda de 2017, del área de residencia, en el área urbana la mayoría de la población, es decir, 18822778 personas (87,9 \%) aprendió en la niñez el idioma castellano y 2344487 (11 \%) manifestaron haber aprendido una lengua autóctona o nativa (quechua, aimara u otra lengua nativa). En el área rural 3386908 (61,8 \%) de la población aprendió el castellano y 2045601 (37,4%) una lengua nativa, especialmente el quechua $(30,3 \%)^{40}$.

El quechua está presente en las 24 regiones del país, siendo las regiones de Apurímac (76,6 \%), Ayacucho (70,6 \%), Huancavelica (66,5\%), Cusco (63,2 \%) y Puno (43,2 \%) las que cuentan con mayor presencia relativa; en el caso de la región Lima representa el 9,5\% de hablantes, y la región de Piura es la que registra la menor tasa relativa (0,2 \%). A su vez, se registra la presencia de aimara hablantes en por lo menos 10 regiones del país, siendo Puno la región que registra la mayor tasa relativa (32,6 \%), seguida de Tacna (20,8 \%) y Moquegua $(12,7 \%)^{41}$. En el caso del quechua, se reconocen dos variantes mayoritarias: quechua sureño (2 395007 hablantes) y quechua central (695 888 hablantes); y dos variantes intermedias: el quechua jauja-huanca (52 788 hablantes) y el quechua norteño (52 203 hablantes).

El número de instituciones educativas que la Dirección Nacional de Educación Bilingüe (DINEBI) considera que son Educación Bilingüe Intercultural es de aproximadamente 3109 en 9 departamentos andinos y de 788 en 6 departamentos de zonas amazónicas ${ }^{42}$ (véanse los cuadros n. ${ }^{\text {os }} 2$ y 3 ).

Cuadro n.․ 2. Número de instituciones educativas EBI - Andes

\begin{tabular}{|l|l|l|}
\hline \multicolumn{1}{|c|}{ Región } & \multicolumn{1}{c|}{ n.o de IE } & \multicolumn{1}{c|}{ Lengua } \\
\hline Ancash & 304 & Quechua variedad Ancash \\
\hline Apurimac & 478 & Quechua variedad Cusco-Collao y Ayacucho Chanka \\
\hline Arequipa & 87 & Quechua variedad Cusco-Collao \\
\hline Ayacucho & 337 & Quechua variedad Ayacucho Chanka \\
\hline Cusco & 648 & Quechua variedad Cusco Collao \\
\hline Huancavelica & 291 & Quechua variedad Ayacucho Chanka \\
\hline Lambayeque & 57 & Quechua variedad Inkahuasi Cañaris \\
\hline Moquegua & 65 & Quechua variedad Cusco Collao y Aymara \\
\hline Puno & 842 & Quechua variedad Cusco Collao y Aymara \\
\hline Total & 3109 & \\
\hline
\end{tabular}

Fuente: Base de datos de la Dirección de Educación Bilingüe Intercultural (2003).

$\overline{40 \text { Instituto Nacional }}$ de Estadística e Informática, Censos Nacionales 2017: XII de Población, VII de Vivienda y III de Comunidades Indígenas (Lima: INEl, 2018), 198.

41 Andrés Chirinos, Atlas lingüístico del Perú (Cusco: Ministerio de Educación - Centro Bartolomé de las Casas, 2001), 23.

42 Lucy Trapnell y Eloy Neira, "Situación de la Educación Intercultural Bilingüe en el Perú" (Lima: Banco Mundial y Programa de Formación en Educación Intercultural Bilingüe - PROEIB-Andes, 2004). https://centroderecursos.cultura. $\mathrm{pe} / \mathrm{sites} /$ default/files/rb/pdf/Situacion\%20de\%20la\%20EBI\%20en\%20el\%20Peru.pdf (04 de abril, 2020) 
Cuadro n.o 3. Número de instituciones educativas EBI - Amazonía.

\begin{tabular}{|l|l|l|}
\hline \multicolumn{1}{|c|}{ Región } & \multicolumn{1}{c|}{ n.o de IE } & \multicolumn{1}{c|}{ Lengua } \\
\hline Cusco & 40 & Machiguenga, Yine, Ashaninka \\
\hline Junín & 166 & Ashaninka, Nomatsiguenga \\
\hline Loreto & 309 & $\begin{array}{l}\text { Guaruna, Chayahuita, Ticuna, Bora, Shipibo, Yine, Kukama, } \\
\text { Kukamiria, Yagua, Candoshi, Huambisa, Achuar, Shapra, } \\
\text { Kichwa, Huitoto, Secoya }\end{array}$ \\
\hline Madre de Dios & 35 & $\begin{array}{l}\text { Amarakaeri, Kichwa, Ese Eja, Shipibo Conibo, Harambukt, } \\
\text { Machiguenga, Amahuaca, Yine }\end{array}$ \\
\hline Pasco & 51 & Ashaninka \\
\hline Ucayali & 187 & Shipibo, Asháninka, Yine,Amahuaca, Machiguenga \\
\hline Total & 788 & \\
\hline
\end{tabular}

Fuente: Base de datos de la Dirección de Educación Bilingüe Intercultural (2003).

\section{La EIB, una utopía hacia una educación de calidad para los pueblos originarios}

A pesar de que se desarrollaron algunas experiencias de educación bilingüe en el Perú antes de la década de 1970, estas no llegaron a formar parte de una política educativa estatal. En realidad, se podría afirmar que la educación bilingüe recién adquirió un respaldo legal en 1972, momento en que se dio la primera Política Nacional de Educación Bilingüe en el marco de la reforma educativa. En la práctica, no obstante, solo se implementaron algunos proyectos experimentales de educación bilingüe que fueron financiados por entidades no gubernamentales y no contaron con una dirección ministerial que los avalara formalmente. Este fue el caso de la experiencia de educación bilingüe de Quinua, llevada a cabo por el Centro de Investigaciones de Lingüística Aplicada (CILA) de la Universidad Nacional Mayor de San Marcos, y del Proyecto Experimental de Educación Bilingüe de Puno (PEEB-Puno), financiado por la GTZ a lo largo de diez años ${ }^{43}$.

En 1991 se creó la Política Nacional de Educación Intercultural y de Educación Bilingüe Intercultural, y en el segundo gobierno de Fujimori (1995-2000) se ordenó la creación de la Unidad Nacional de Educación Bilingüe Intercultural sin nivel de dirección, decisión tomada debido a la presión del Banco Mundial, que exigía que el Perú tuviera una política de Educación Bilingüe Intercultural como condición para financiar los proyectos de desarrollo, y a los memoriales y reclamos de los entendidos en la materia y de algunos maestros. Desde el año 2002, esta Unidad Nacional de Educación Bilingüe Intercultural del Ministerio de Educación se convirtió en Dirección Nacional y a partir de este cambio la EBI pasó a ser una política educativa del Estado.

43 Virginia Zavala y Gavina Córdova. "Volver al desafío: Hacia una definición crítica de la educación bilingüe intercultural en el Perú" (Lima: Proeduca, GTZ, 2003), 7. 
De acuerdo con la publicación de resultados y el análisis acerca del aprendizaje y los rendimientos escolares de los últimos años en el Perú, el principal rasgo de realidad que emerge es que hay un rendimiento muy bajo y también una gran desigualdad; esto último determina que la gran cobertura existente sea menos valiosa y que el escaso presupuesto asignado se convierta en un factor importante para explicar el estado de la educación en el país ${ }^{44}$.

Las políticas educativas impartidas en la sociedad peruana han incidido definitivamente en las gravísimas dificultades que enfrenta, con más de $90 \%$ de estudiantes de $2 .^{\circ}$ y $6 .{ }^{\circ}$ grados de primaria que no han logrado un desarrollo óptimo de las capacidades matemáticas más elementales demandadas por el currículo; el $85 \%$ y $88 \%$ de los estudiantes de $2 .^{\circ}$ y $6 .{ }^{\circ}$ grados de primaria, respectivamente, que no han desarrollado las habilidades básicas para leer de manera comprensiva ni cuentan con las herramientas necesarias para continuar su proceso de alfabetización. El 94 \% y 97,1 \% de los estudiantes de 3. ${ }^{\circ}$ y 5..$^{\circ}$ de secundaria, respectivamente, muestran limitaciones para reflexionar, realizar inferencias y para comprender y resolver las situaciones de contenido matemático elemental que se les presentan ${ }^{45}$.

Los siguientes indicadores configuran claramente el actual panorama de crisis de la educación en el Perú: la Evaluación Nacional del 2004 muestra que el nivel alcanzado por los estudiantes peruanos es preocupante y, lo que es peor, no ha mejorado respecto al nivel registrado en las evaluaciones anteriores de 1996, 1998 y 2001. Mirando lo que sucede al analizar el nivel primario, encontramos que solo $12,1 \%$ de los estudiantes logran los aprendizajes esperados en comprensión de textos y el 9,6 \% en matemática; es decir, alrededor de $90 \%$ de los estudiantes ingresan a la secundaria con conocimientos insuficientes. Así, al terminar la secundaria, los resultados son aún peores: 9,8 \% de alumnos alcanzan lo esperado en comprensión de textos y 2,9 \% en matemática (Unidad de Medición de la Calidad del Ministerio de Educación, 2005) ${ }^{46}$.

Según los datos estadísticos oficiales del INEI, Perú tiene un 5,9 \% de tasa de analfabetismo en la población mayor de 15 años, agravándose esta situación en las áreas rurales, donde representa un 14,9\% frente al 3,5\% de las áreas urbanas. En las comunidades que tienen como lengua materna una nativa (quechua, aimara o lenguas amazónicas) el analfabetismo es del 16,1\%. Si se cruzan estos datos con la variable sexo, se tiene que entre los varones mayores de 15 años el analfabetismo es de 6,6\%, y las mujeres conforman el sector más marginado ya que triplican el porcentaje, con $25,3 \%{ }^{47}$.

En el marco de la diversidad, la política de EIB exige nuevos desafíos en cuanto a la aplicación del programa, que no solo merece una revisión objetiva y detallada sino que exige acciones en las formas de enseñar y aprender lenguas ${ }^{48}$; los recursos y programas sociales

44 Roxana Barrantes y Javier Iguiñiz. La investigación económica y social en el Perú. Balance 1999-2003 y prioridades para el futuro (Lima: Consorcio de Investigación Económica y Social, 2004), 158.

45 Hugo Díaz y León Trahtemberg. "Plan de acción inmediata para la Educación Escolar", en Perú, ¿en qué país queremos vivir?: la apuesta por la educación y la cultura, vol. III (Lima: CADE, IPAE, 2001), 47.

46 Martínez Alpaca. "El enfoque del Estado en las políticas educativas interculturales bilingües", 87.

47 Sonia García Segura. "Identidad, lengua y educación: la realidad de la amazonía peruana", Revista de estudios y experiencias en educación vol. 18, n. ${ }^{\circ} 36$ (2019): 201.

48 Yamith José Fandiño Parra et al. "Retos del Programa Nacional de Bilingüismo. Colombia Bilingüe", Revista Educación y Educadores vol. 15, n. ${ }^{\circ} 3$ (2012): 373. 
del gobierno orientados a combatir la pobreza, reducir las desigualdades socio- económicas y ampliar las oportunidades de la población indígena han probado ser insuficientes para proveer de mejores oportunidades de desarrollo humano ${ }^{49}$. Por ello, la Educación Intercultural Bilingüe se visualiza como un proyecto político-pedagógico que busca la autonomía y el fortalecimiento cultural de los pueblos para que puedan contribuir, desde un diálogo simétrico, a la cultura y el desarrollo del conjunto de la sociedad y los territorios ${ }^{50}$.

La educación bilingüe y la interculturalidad son instrumentos revolucionarios y, por tanto, imprescindibles para toda sociedad heterogénea y plural, porque aquella permite la apropiación de las actitudes, conocimientos y destrezas por dos vías lingüísticas, y esta nos conduce al respeto de la identidad étnica de los grupos involucrados ${ }^{51}$. La EIB, en este contexto, exige un análisis y replanteamiento de estrategias en los aspectos político y pedagógico, ya que la política trasciende lo educativo para pensar en la construcción de sociedades diferentes. Como decía Paulo Freire, "la educación intercultural en sí solo tendrá significación, impacto y valor cuando esté asumida de manera crítica, como acto pedagógico-político que procura intervenir en la refundación de la sociedad" 52 . En tal sentido, el contacto y las relaciones entre los pueblos indígenas, afrodescendientes, blanco-mestiza y criolla, evidencian la necesidad de fortalecer la política de EIB en el Estado peruano.

Los pueblos indígenas han transmitido de forma oral, por generaciones, sus saberes y conocimientos en campos diversos como la botánica, la medicina, la agricultura, la astronomía, etc., erudición y experiencia que, a falta de una sistematización escrita, corren el riesgo de perderse junto con las lenguas que las han comunicado. De ocurrir tal extinción la humanidad perdería recursos incalculables producidos por la práctica y el desarrollo del conocimiento humano a lo largo de los siglos. Desdichadamente, aún no existe tanta sensibilidad frente a la muerte lingüística como a la de especies animales y vegetales ${ }^{53}$.

El Estado peruano es un país pluriétnico, multicultural y plurilingüe, que se manifiesta en la coexistencia de diversos pueblos andinos, amazónicos y costeños donde, "(...) se reconoce la diversidad existente, vista desde una óptica de la cultura dominante y nacional, pero lejos de la utopía de la intersección, interpenetración o interestructuración positiva de culturas"s54.

(...) la propuesta intercultural, que pareciera ser una vía efectiva de reconocimiento del "otro", un modo de desheredarse del etnocentrismo y abrirse a la valoración de conocimientos ancestrales propios, está aún pendiente, y en ese sentido constituye una utopía para la educación en su conjunto y para la escuela como invención moderna, en singular. ${ }^{55}$

49 Martha Singer Sochet. "¿Exclusión o inclusión indígena?", Estudios Políticos, n. 31 (2011): 91.

50 Williamson y Navarrete. "Cooperación internacional y Educación Intercultural Bilingüe", 25.

51 Vicente Santiváñez Limas. "La educación bilingüe intercultural y la identidad nacional", Revista Cultura, n. 23 (2009): 102.

52 Paulo Freire citado en Walsh. "Interculturalidad crítica y educación intercultural", 1.

53 Luis Enrique López y Wolfgang Küper. "La educación intercultural bilingüe en América Latina: balance y perspectivas", Revista Iberoamericana de Educación vol 20 (1999): 23.

54 F. Garcés y A. Manco citados en Elizabeth Castillo Guzmán y Sandra Patricia Guido Guevara. "La interculturalidad: ¿principio o fin de la utopía?". Revista Colombiana de Educación, n. ${ }^{\circ} 69$ (2015): 40 [cursivas del original].

55 Castillo Guzmán y Guido Guevara. "La interculturalidad: ¿principio o fin de la utopía?", 27. 
En Perú, la dificultad y la fortuna empiezan ahí, en la inmensa meta-biodiversidad de rostro humano. El proyecto de la EIB es, sin discusión alguna, una propuesta clara en la que los elementos culturales congenian entre los cuerpos y lenguajes de mundos diferentes en condiciones desiguales. Esta viene a ser una de las más difíciles tareas que tiene el gobierno como plan piloto al bicentenario (2021), y a un proyecto global sostenible al 2030. Desarrollar y refundar políticas públicas sobre la base de una educación intercultural que permita reconocer la inmensa diversidad étnico-lingüística es una entre muchas alternativas, pero una de las más eficientes en integrar saberes y sentires que puedan recomponer el equilibrio con la naturaleza y la institución de un nuevo modelo de ciudadanía ${ }^{56}$.

El Perú cuenta con un Plan Nacional de Educación Bilingüe al 2021 con la finalidad de garantizar aprendizajes pertinentes y de calidad a niños, niñas, adolescentes, jóvenes, personas adultas y personas adultas mayores de todo el Perú, pertenecientes a los pueblos indígenas u originarios que contribuyan a la formación de ciudadanos y ciudadanas protagonistas en la construcción de un proyecto colectivo de sociedad inclusiva, democrática y plural con igualdad de género. El objetivo es brindar un servicio educativo relevante y pertinente, que garantice la mejora de los aprendizajes de los niños, niñas, adolescentes, jóvenes, personas adultas y personas adultas mayores pertenecientes a los pueblos originarios a través de la implementación de una educación intercultural y bilingüe en todas las etapas, formas y modalidades del sistema educativo, desde una perspectiva crítica de tratamiento de la diversidad étnica, cultural y lingüística del país. PNEB al 2021 cuenta con finalidades y objetivos trazados, pero no se han logrado superar los aprendizajes esperados en los pueblos originarios, que se convierten en una utopía hacia una educación de calidad.

\section{Conclusiones}

Las políticas de educación bilingüe del siglo XX y lo que va corrido del XXI han estado determinadas no solo por las condiciones económicas, políticas y sociales propias del Estado peruano, sino por las influencias y condicionamientos de los organismos multilaterales FMI -BM y el positivismo cientificista. De igual manera, la globalización es un proceso que influye de manera determinante en todos los grupos y actores sociales y, en este sentido, los pueblos indígenas no están libres de su influencia. La educación bilingüe de calidad es un desafío para los pueblos indígenas y es saber cómo enfrentarlo e identificar cuáles son las dificultades y las oportunidades que tienen ante un fenómeno social, político y económico que surge la necesidad de lograr desde la escuela y la comunidad actitudes transformadoras y de construcción de un Estado diferente, pluricultural e incluyente, con la posibilidad de una nueva sociedad o Estado con respeto ante la diversidad étnica, cultural y lingüística.

Perú hacia el bicentenario contempla una vasta diversidad etnolingüística; los datos del Ministerio de Cultura refieren que en el Perú existen 55 pueblos originarios o indígenas que hablan 47 lenguas originarias, 43 amazónicas y 4 andinas, lenguas que se consideran vigentes porque tienen hablantes, y asimismo una diversidad étnica, social, cultural y biológica que determina diversos modos de ser, de hablar, de sentir, de pensar. Por la diversidad

56 Canaza-Choque y Huanca-Arohuanca. "Perú 2018: hacia una Educación Intercultural Bilingüe sentipensante", 520.

Rev. hist.edu.latinoam - Vol. 23 No. 36, enero - junio 2021 - ISSN: 0122-7238 - pp. 205 - 225 
etnolingüística la educación del siglo XXI es prioritario que las lenguas y culturas originarias sean entendidas como medio válido de aprendizaje porque son poseedoras de saberes y conocimientos referidos a distintos campos, como la botánica, la medicina, la agricultura, gastronomía, entre otros.

La educación bilingüe adquirió, tardíamente, respaldo legal en 1972, momento en el que se produjo la primera Política Nacional de Educación Bilingüe en el marco de la reforma educativa del gobierno de Juan Velasco Alvarado, y en la segunda fase (1975-1980) del gobierno militar del general Francisco Morales Bermúdez, quien difundió el documento "Lineamiento de la Política Educativa Nacional" con el objetivo de anular la reforma educativa hecha por su antecesor. En el segundo gobierno de Fujimori (1995-2000) se ordenó la creación de la Unidad Nacional de Educación Bilingüe Intercultural sin nivel de dirección, decisión tomada debido a la presión del Banco Mundial como condición para financiar los proyectos de desarrollo. En la actualidad, el principal rasgo de la realidad que emerge en la implementación de la educación bilingüe es que los estudiantes se encuentran en un rendimiento no esperado, en el nivel muy bajo de acuerdo con los estándares de evaluación y también se encuentran una gran desigualdad, en tal sentido la educación bilingüe de calidad para los pueblos originarios se convierte en una utopía.

\section{Conflicto de interés}

El autor no tienen conflicto de interés.

\section{Financiamiento}

El trabajo de investigación es autofinanciado.

\section{Referencias}

Asamblea Constituyente, Perú 1979. Constitución Política del Perú 1979. Lima: ACP, 1979.

Barrantes, Roxana y Javier Iguiñiz. La investigación económica y social en el Perú. Balance 1999-2003 y prioridades para el futuro. Lima: Consorcio de Investigación Económica y Social, 2004.

Canaza-Choque, Franklin y Jesús Huanca-Arohuanca. "Perú 2018: hacia una Educación Intercultural Bilingüe sentipensante". Sciéndo. Ciencia para el desarrollo, 21, n.o 4 (2018): 515-522. https://doi.org/10.17268/sciendo.2018.058

Castillo Guzmán, Elizabeth y Sandra Patricia Guido Guevara. “La interculturalidad: ¿principio o fin de la utopía?". Revista Colombiana de Educación, no 69 (2015): 17-44. https://doi.org/10.17227/01203916.69rce17.44

Ccahuana Córdova, Jorge Alberto. "Según la capacidad intelectual de cada uno: élites, Estado y educación indígena a inicios del siglo XX”. Tesis de licenciatura en Historia, Pontificia Universidad Católica del Perú, 2013.

Chirinos, Andrés. Atlas lingüístico del Perú. Cusco: Ministerio de Educación - Centro Bartolomé de las Casas, 2001. 
Díaz, Hugo y León Trahtemberg. “Plan de acción inmediata en Educación Escolar". En Perú, ¿en qué país queremos vivir?: la apuesta por la educación y la cultura, vol. III. Lima: CADE, IPAE, 2001.

Dulzaides, María Elinor y Ana María Molina. "Análisis documental y de información: dos componentes de un mismo proceso". Acimed 12, n.o 2 (2004): 8-12.

Enrique López, Luis y Wolfgang Küper. "La educación intercultural bilingüe en América Latina: balance y perspectivas". Revista Ibero Americana de Educación 20 (1999): 17-85. https://doi.org/10.35362/rie2001041

Espinosa, Oscar. "Los planes de vida y la política indígena en la Amazonía peruana". Anthropologica 32, n. 32 (2014): 87-114.

Fajardo Salinas, Delia María. "Educación intercultural bilingüe en Latinoamérica: un breve estado de la cuestión". LiminaR. Estudios sociales y humanísticos IX, n.o 2 (2011): 15-29. https://doi.org/10.29043/liminar.v9i2.45

Fandiño Parra, Yamith José, Jenny Raquel Bermúdez Jiménez y Víctor Elías Lugo Vásquez. “Retos del Programa Nacional de Bilingüismo. Colombia Bilingüe". Revista Educación y Educadores 15, n.03(2012):363-381. https://doi.org/10.5294/edu.2012.15.3.2

Ferrão Candau, Vera María. "Educación intercultural en América Latina: distintas concepciones y tensiones actuales". Estudios Pedagógicos 36, n.o 2 (2010): 333-342. https://doi.org/10.4067/S0718-07052010000200019

García Segura, Sonia. "Identidad, lengua y educación: la realidad de la amazonía peruana". Revista de estudios y experiencias en educación 18, n.o 36 (2019): 193-207. https://doi.org/10.21703/rexe.20191836garcial

Godenzzi, Juan Carlos. “La educación bilingüe intercultural”. Lexis XXV, n.os 1 y 2 (2001): 299-318.

Grimaldo Muchotrigo, Miriam. “Identidad y política cultural en el Perú". Liberabit, n.o 12 (2006): 41-48.

Huamán Cosi, Melchor. “Educación Bilingüe Intercultural (EBI) en el Perú, siglo XXI”. Perú: Lanic, 2007. http://lanic.utexas.edu/project/etext/liilas/ilassa/2008/cosi.pdf. (30/3/2020).

Instituto Nacional de Estadística e Informática. Censos Nacionales 2017: XII de Población, VII de Vivienda y III de Comunidades Indigenas. Lima: INEI, 2018.

Instituto Nacional de Desarrollo de Pueblos Andinos, Amazónicos y Afroperuanos (INDEPA). "Mapa Etnolingüístico del Perú". Revista Peruana de Medicina Experimental y Salud Pública vol. 27, n.o 2 (2010): 288-291. https://doi.org/10.1590/S1726-46342010000200019

Instituto Nacional de Estadística e Informática. “Evolución de la pobreza monetaria 2009-2014. Informe Técnico". Lima, 2015.

Knobel, Michele y Colin Lankshear. Maneras de saber: tres enfoques para la investigación educativa. Morelia, México: Universidad Pedagógica Nacional, 2003.

López, Luis Enrique. "La cuestión de la interculturalidad y la educación latinoamericana". Documento de apoyo. Séptima reunión del Comité Regional Intergubernamental del Proyecto Principal de Educación en América Latina y el Caribe Cochabamba, 5 al 7 de marzo de 2001.

Martínez Alpaca, Ynty Koyllor. “El enfoque del Estado en las políticas educativas interculturales bilingües: Los planes educativos en el Perú, 2005-2008". Tesis de maestría en Política Social, Universidad Nacional Mayor de San Marcos, 2012.

Ministerio de Educación. Política Nacional de Educación Bilingüe. Lima: MINEDU, 1972.

Ministerio de Educación. Documento nacional de Lenguas originarias del Perú. Lima: MINEDU, 2013.

Ministerio de Educación. Hacia una Educación Intercultural Bilingüe de Calidad. Lima: MINEDU, 2013.

Ministerio de Educación. Lenguas originarias del Perú. Lima: MINEDU, 2013.

Ministerio de Educación. Política sectorial de Educación Intercultural y Educación Intercultural Bilingüe. Lima: MINEDU, 2018. 
Mondaca Rojas, Carlos y Yeliza Gajardo Carvajal. "La Educación Intercultural Bilingüe en la región de Arica y Parinacota, 1980-2010, 1980-2010". Diálogo Andino n.o 42 (2013): 69-87. https://doi.org/10.4067/S0719-26812013000200007

Paredes, David E., Diego Vera, Karlos La Serna y Wilfredo Barrionuevo. “Diagnóstico de la Educación Intercultural Bilingüe (EIB) en el Perú". Informe de la Universidad de San Martín de Porres, 17 de marzo de 2017. https://usmp.edu.pe/idp/wp-content/uploads/2016/05/diagnstico_de_eib_en_el_per. pdf. $(30 / 3 / 2020)$

Pulido, Yeny Alexandra. "La etnoeducación bilingüe: logro político y desafío para las etnias". Lenguaje 40, n.o 1 (2012): 231-254. https://doi.org/10.25100/lenguaje.v40i1.4950

Ruelas Vargas, David. "Los movimientos indígenas y la educación del siglo XX en el sur andino puneño peruano". Revista Historia de la Educación Latinoamericana 21, n.o 33 (2019): 61-85. https://doi. org/10.19053/01227238.9354. https://doi.org/10.19053/01227238.9354

Santiváñez Limas, Vicente. "La educación bilingüe intercultural y la identidad nacional”. Revista Cultura, n.ㅇ 23 (2009): 101-114.

Schmelkes, Sylvia. "Educación para un México intercultural". Sinéctica, n.o 40 (2013): 1-12.

Singer Sochet, Martha. “¿Exclusión o inclusión indígena?”. Estudios Políticos, n.o 31, (2011): 87-106. https://doi.org/10.1016/S0185-1616(14)70572-4

Tamayo y Tamayo, Mario. Módulo 2. La investigación. Serie Aprender a investigar. Santa Fe de Bogotá: ICFES, 1998.

Trapnell, Lucy y Eloy Neira. "Situación de la Educación Intercultural Bilingüe en el Perú". Lima: Banco Mundial y PROEIB-Andes, 2004. https://centroderecursos.cultura.pe/sites/default/files/rb/pdf/ Situacion\%20de\%20la\%20EBI\%20en\%20el\%20Peru.pdf.

Valiente-Catter, Teresa. "La educación intercultural bilingüe en la encrucijada global. Currículo intercultural y aprendizaje multicultural". Alteridad. Revista de Educación 3, n.o 2 (2008): 24-43. https://doi.org/10.17163/alt.v3n2.2008.02

Vásquez Huamán, Enrique, Annie Chumpitaz y César Jara. Niñez indígena y Educación Intercultural Bilingüe en el Perú: Estadísticas recientes, preguntas (i) resueltas y tareas pendientes. Lima: Care Perú, 2009.

Walsh, Catherine. "Interculturalidad crítica y educación intercultural". En Construyendo Interculturalidad Crítica, editado por J. Viaña, L. Tapia y C. Walsh. La Paz: Instituto Internacional de Integración del Convenio Andrés Bello, 2010.

Williamson, Guillermo y Susana Navarrete. “Cooperación Internacional y Educación Intercultural Bilingüe en Chile". Revista Mexicana de Investigación Educativa 19, n.o 60 (2014): 19-43.

Zavala, Virginia y Gavina Córdova. "Volver al desafío: Hacia una definición crítica de la educación bilingüe intercultural en el Perú". Lima: Proeduca, GTZ, 2003.

Zúñiga Castillo, Madeleine. La Educación Intercultural Bilingüe: el caso peruano. Buenos Aires: Foro Latinoamericano de Políticas Públicas - FLAPE, 2008.

Zúñiga Castillo, Madeleine y Juan Ansión Mallet. Interculturalidad y educación en el Perú. Lima: Foro Educativo, 1997. https://red.pucp.edu.pe/ridei/wp-content/uploads/biblioteca/inter59.PDF 